\title{
Claudin expression in high-grade invasive ductal carcinoma of the breast: correlation with the molecular subtype
}

Shaolei Lu, Kamaljeet Singh, Shamlal Mangray, Rose Tavares, Lelia Noble, Murray B Resnick and Evgeny Yakirevich

Department of Pathology, Rhode Island Hospital, and Alpert Medical School of Brown University, Providence, RI, USA

\begin{abstract}
Claudin proteins are a major component of the tight junctions. Dysregulation of claudin protein expression has been described in a number of malignancies. Gene expression profiling has stratified breast cancers into distinct molecular subtypes: luminal, HER2 positive (HER2 +), and basal-like. Recently, a novel claudin-low molecular subtype has been described. In this study, we correlated the expression patterns of claudins with the molecular subtypes of breast cancer. On the basis of immunohistochemical expression, 226 grade 3 invasive ductal carcinomas were stratified into 65 luminal (estrogen receptor positive (ER + )), 65 HER2 +, 86 basal-like, including 14 metaplastic carcinomas (ER - , HER2 - , CK5/6, and/or epidermal growth factor receptor positive), and 10 unclassified. Tissue microarrays were analyzed for the expression of claudins 1, 3, 4, 7, and 8 by immunohistochemistry and scored semiquantitatively. High levels of expression were detected in $17 \%$ of all cases for claudin $1,32 \%$ claudin $3,41 \%$ claudin $4,44 \%$ claudin 7 , and $40 \%$ claudin 8 . Luminal cancers exhibited increased claudins 7 and 8; basal-like tumors demonstrated increased expression of claudins 1 and 4 . Low expression of all five claudins was detected in 30 of 226 cases $(13 \%)$ and this group was designated 'claudinlow'. The majority of the claudin-low subgroup were basal-like cancers ( 23 of $30,77 \%)$. In contrast, only 1 of 30 $(3 \%)$ claudin-low tumors was of the luminal phenotype and 6 of 30 cases $(20 \%)$ were HER2 $+(\boldsymbol{P}<0.001)$. Within the basal-like subgroup, $64 \%$ of the metaplastic and $19 \%$ of the non-metaplastic tumors were claudin-low. The claudin-low group was strongly associated with disease recurrence $(P=0.0093)$. In conclusion, this study is the first to examine comprehensively the differential expression of claudins 1, 3, 4, 7, and 8 in the molecular subtypes of high-grade breast cancer. Claudin-low subtype is a frequent phenomenon in metaplastic and basallike breast cancer and appears to be a strong predictor of disease recurrence.

Modern Pathology (2013) 26, 485-495; doi:10.1038/modpathol.2012.187; published online 7 December 2012
\end{abstract}

Keywords: breast; carcinoma; claudin

Breast cancer is the most common type of female cancer and represents $14 \%$ of cancer-related deaths in women. ${ }^{1}$ It has been estimated that there will be about 226870 new cases of breast cancer in the United States in 2012, and about 39510 women will die from this disease. ${ }^{1}$ Breast cancer is a genetically heterogeneous group of tumors with a variety of morphological features. Global gene expression

Correspondence: Dr S Lu, MD, PhD, Department of Pathology, Rhode Island Hospital, APC-12, 593 Eddy Street, Providence, RI 02903, USA.

E-mail: slu@lifespan.org

This study was presented in part at the annual meeting of the United States and Canadian Academy of Pathology in San Antonio, TX in 2011.

Received 19 May 2012; revised 6 September 2012; accepted 14 September 2012; published online 7 December 2012 profiling studies have produced a new molecular classification of breast cancer with four distinct subtypes: luminal, HER2, basal-like, and unclassified. ${ }^{2,3}$ Each of these subtypes has unique biological and prognostic features. As microarray gene expression analysis is not routinely available, immunohistochemical surrogates have been developed, which approximate the molecular subtypes of breast cancer. ${ }^{4,5}$ Based on an immunohistochemical panel of four markers (estrogen receptor (ER), HER2, epidermal growth factor receptor (EGFR), and cytokeratin 5/6), breast cancers may be stratified into ER + /HER2 - (luminal), HER2 + / any ER (HER2-enriched), ER - /HER2 - /EGFR + and/or cytokeratin 5/6+ (basal-like), and ER - / HER2 - /EGFR - /cytokeratin 5/6 - (unclassified). ${ }^{4}$ Recent gene expression studies have identified a 
novel claudin-low subtype of breast cancer. ${ }^{6}$ This claudin-low subgroup has been proposed to be related to metaplastic cancer, epithelialmesenchymal transition, and poor prognosis. ${ }^{7-9}$

Claudins are members of a large family of tight junction proteins, which regulate cellular adhesion, polarity, and glandular differentiation. There are currently at least 24 different claudins known to exist in humans, and the expression of each appears to be tissue specific. ${ }^{10,11}$ Claudin expression is frequently altered in several cancers. ${ }^{12-14}$ Because of the high specificity of claudin expression patterns in cancer tissue, it has been proposed that claudins may represent useful diagnostic and prognostic markers in gastrointestinal, genitourinary, and pulmonary neoplasms. ${ }^{15-21}$

Comparatively few studies have examined the expression of claudins in breast cancer. Claudin 7 has been reported to be downregulated in high-grade invasive ductal carcinomas, in the ER - and basallike molecular subtypes. ${ }^{22-24}$ In contrast, claudins 3 and 4 have been found to be overexpressed in breast cancer at both the mRNA and protein level..$^{25-28}$

In this study, we examined the expression of claudins 1, 3, 4, 7, and 8 in a cohort of patients with grade 3 breast carcinoma representing the different molecular subtypes of breast cancer to identify and better characterize the claudin-low subgroup.

\section{Materials and methods}

\section{Tissue Selection}

Tissue samples of grade 3 invasive ductal carcinoma from 226 consecutive patients aged 27-96 years were collected between the years 1996 and 2009 from the archives of the Departments of Pathology at the Rhode Island Hospital and The Miriam Hospital. The cohort of grade 3 tumors was selected based on the fact that claudin-low breast carcinomas are mainly histologically grade 3 tumors. ${ }^{9}$ None of these patients received neoadjuvant chemotherapy or radiotherapy before surgery. After surgery, 100 (44\%) patients received chemotherapy alone, $20(9 \%)$ received hormonal therapy alone, and $36(16 \%)$ received both chemotherapy and hormonal therapy. This study was approved by the institutional review board at the Rhode Island Hospital. The histological grade was previously determined according to the Nottingham modification of the Bloom-Richardson scoring system $^{29}$ and confirmed independently by two pathologists (EY and KS). Stage of disease was defined according to the American Joint Committee on Cancer (AJCC). ${ }^{30}$ Based on the immunohistochemical expression of ER, HER2, EGFR, and cytokeratin 5/6, the tumors were stratified into 65 luminal (ER + /HER2 - /any EGFR and/or cytokeratin 5/6), 65 HER2-enriched (HER2 + /any ER), 86 basallike (ER - , HER2 - , CK5/6, and/or EGFR + ), and 10 unclassified (ER - /HER2 - /EGFR - /cytokeratin 5/6 - ) according to Nielsen et al. ${ }^{4}$ The basal-like group included 14 metaplastic carcinomas, of which 6 had a squamous component, 5 spindle sarcomatous areas, and 7 with heterologous elements (5 chondroid and 2 osseous). Four tumors contained two or more components.

\section{Tissue Microarray Construction}

Paraffin blocks containing representative tumor areas were identified on corresponding hematoxylin- and eosin-stained sections. Areas of interest were identified and marked on the source block. The source block was cored, and a 1-mm core was transferred to the recipient 'master block' using the Beecher Tissue Microarrayer (Beecher Instruments, Silver Spring, MD, USA). Five representative cores of tumor and two cores of normal breast tissue were arrayed per specimen.

\section{Immunohistochemical Staining}

Immunohistochemical staining was performed according to the following protocol. Sections from paraffin-embedded tissue microarrays were cut at $4 \mu \mathrm{m}$, deparaffinized, and rehydrated with xylene and graded alcohols. Microwave epitope retrieval was performed in target retrieval ( $\mathrm{pH} \mathrm{6.0)}$ (Dako, Carpinteria, CA, USA) for ER, and HER2; high pH target retrieval for cytokeratin 5/6 (Dako); or $10 \mathrm{mM}$ citrate buffer $(\mathrm{pH}$ 6.0) for $10 \mathrm{~min}$, followed by cooling for $15 \mathrm{~min}$ at room temperature for claudins. The following primary antibodies were used: clone ER1D5 against ER (1:300 dilution; Dako), clone CB11 against HER2 (1:150 dilution; Vector Laboratories), clone D5/16B4 against cytokeratin 5/6 (1:40 dilution; Cell Marque, Rocklin, CA, USA), rabbit polyclonal antibodies against claudin 1 (1:50 dilution; NeoMarkers, Lab Vision, Fremont, CA, USA), claudin 3 (1:1000; NeoMarkers), claudin 4 (1:1000; NeoMarkers), claudin 7 (1:500; NeoMarkers), and claudin 8 (1:100; GeneTex, San Antonio, TX, USA). Appropriate positive and negative controls were used simultaneously with test slides. Positive controls consisted of normal colonic mucosa. ${ }^{15}$ Normal rabbit serum (sc-2338; Santa Cruz Biotechnology, Santa Cruz, CA, USA) was used as the negative control for anti-claudin antibodies.

Immunohistochemical staining for ER and HER2 was performed using the Dako Autostainer Plus and EnVision Dual Link detection reagent (Dako) with DAB (Dako). Immunohistochemical staining for claudins 3, 4, 7, and 8 was performed using the Envision Plus kit (Dako) and for claudin 1 using the Ventana Discovery automated staining system employing the Ventana Basic DAB detection kit and endogenous biotin-blocking kit (Ventana Medical Systems, Tucson, AZ, USA). EGFR was stained using the PharmDX kit (Dako) according to the manufacturer's instructions. For HER2 fluorescent 
in situ hybridization assay, slides were hybridized with probes to LSI HER2/neu and CEP 17 with the PathVysion HER2 DNA Probe Kit (Abbott Molecular, Des Plaines, IL, USA) according to the manufacturer's instructions. Slides were counterstained with 4',6-diamidino-2-phenylindole and visualized on a Zeiss Axioplan epifluorescent microscope (Zeiss, Baden-Wurttemberg, Germany).

Staining results were assessed by two pathologists (EY and SL) in a blinded manner. ER stains were considered positive if immunostaining was seen in more than $1 \%$ of tumor nuclei. EGFR stains were considered positive if any (weak or strong) membranous invasive carcinoma cell staining was observed. CK5/6 stains were scored positive if any (weak or strong) cytoplasmic and/or membranous staining was detected in the tumor cells. For HER2 status, tumors were considered positive if scored as $3+$ according to the guidelines of the American Society of Clinical Oncology/College of American Pathologists, ${ }^{31}$ and fluorescent in situ hybridization with amplification ratio 2.2 or more was used to segregate immunohistochemically equivocal $(2+)$ results. Claudin immunoreactivity was assessed based on a combined score of the extent and intensity of staining. Scores 0-3 were assigned according to the percentage of positive tumor cells $(0=0 \%$; $1=<25 \% ; 2=25-50 \%$; and $3=>51 \%$ ) and the intensity of staining in tumor $(0=0 ; 1=1+$; $2=2+$; and $3=3+$ ) as described previously. ${ }^{18}$ The two scores were multiplied to give an overall score of $0-9$, of which 0 was considered negative, 1-2 was considered weak, 3-6 moderate, and 9 strong staining. ${ }^{16}$ Negative and weak expression was considered as low, whereas moderate and strong as high. Tumors with low expression of all five claudins were defined as claudin-low. Any discordant scores were reviewed together by both scorers to obtain a consensus score.

\section{Statistical Methods}

The $\chi^{2}$ analysis was used to assess the associations between the expression of claudins and molecular subtypes. Hierarchical clustering was used to detect the association between claudins and ER, HER2, EGFR, and CK5/6 expression by centroid method with data standardization. Complete method with data standardization was used for hierarchical clustering of histological features of claudin-low breast cancer. Overall survival time was calculated from the time of diagnosis until the time of death. The method of Kaplan-Meier was used to generate overall survival curves, and curves were compared using a log-rank test. The prognostic significance of claudin expression and the other clinical variables, including tumor size, lymph node status, presence of metastasis, and AJCC stage, was determined using a univariate Cox proportional hazards model. Multivariate analysis was carried out using a multivariate
Cox proportional hazards model, including the following variables: age, claudin expression, tumor molecular subtype, AJCC stage, and systemic adjuvant therapy. All tests were two-sided with 0.05 as the threshold to be considered statistically significant. All analyses were performed using the SAS software, JMP Base version 8.0.0 (SAS, Cary, NC, USA).

\section{Results}

\section{Clinicopathological Characteristics}

The clinicopathological characteristics of the patients with grade 3 invasive ductal carcinomas are summarized in Table 1. In all, 102 patients (45\%) presented with T2 or larger tumors; 71 (31\%) with nodal involvement; and $6(3 \%)$ with metastatic disease. A total of 26 patients (12\%) had advanced stage $^{3-4}$ disease at diagnosis (defined according to the AJCC). Clinical follow-up was available for all cases. Median follow-up was 52.5 months (range, 1-211.6 months).

\section{Expression of Claudins in Breast Cancer}

The normal breast ductal and lobular epithelium expressed claudins 1, 3, 4, 7, and 8 (Figure 1). In normal breast tissue, all of the claudins exhibited predominantly an apicolateral membranous staining pattern of luminal cells with no expression by myoepithelial cells. In invasive ductal carcinoma cases, claudin 1 demonstrated a predominantly punctate membranous staining pattern, whereas claudins 3, 4, 7, and 8 staining varied from a punctate to complete circumferential membranous staining with no preservation of the apicolateral polarity seen in normal breast epithelium.

High levels of expression were detected in $17 \%$ of all tumors for claudin 1, 32\% claudin 3, 41\% claudin $4,44 \%$ claudin 7 , and $40 \%$ for claudin 8 . The frequency of high-level claudin 1 expression was approximately $50 \%$ that of other claudin types. Based on the protein expression of claudins, ER, HER2, CK5/6, and EGFR, a hierarchical clustering analysis was performed and the results provided a global overview of the relationship among these markers (Figure 2). Cases with high expression of claudins 1,3 , and 4 clustered in one group with cases positive for basal markers CK5/6 and EGFR. In contrast, tumors with high expression of claudins 7 and 8 clustered with ER + neoplasms. HER2 + tumors did not cluster with any of claudins.

While cluster analysis provided a comprehensive overview, correlation analysis revealed significant association between these markers (Table 2). When tumors were categorized into low (negative and weak expression) and high (moderate and strong expression) for each claudin, there were strong positive associations between claudin 1 and CK5/6 
Table 1 Clinicopathological characteristics of patients with different molecular subtypes of breast carcinoma

\begin{tabular}{|c|c|c|c|c|c|}
\hline \multirow{2}{*}{ Parameter } & \multirow{2}{*}{ Total } & \multicolumn{4}{|c|}{ Molecular subtype } \\
\hline & & Luminal & HER2 & Basal-like & Unclassified \\
\hline Sample size (\%) & 226 & $65(29 \%)$ & $65(29 \%)$ & $86(38 \%)$ & $10(4 \%)$ \\
\hline Mean age (years) \pm s.d. & $61 \pm 15$ & $64 \pm 16$ & $61 \pm 15$ & $59 \pm 15$ & $65 \pm 12$ \\
\hline \multicolumn{6}{|l|}{ Race } \\
\hline White & $202(90 \%)$ & $62(95 \%)$ & $59(92 \%)$ & $73(85 \%)$ & $9(90 \%)$ \\
\hline African American & $24(10 \%)$ & $3(5 \%)$ & $5(8 \%)$ & $13(15 \%)$ & $1(10 \%)$ \\
\hline \multicolumn{6}{|l|}{ AJCC stage } \\
\hline Stage 1 & $91(41 \%)$ & $20(31 \%)$ & $30(46 \%)$ & $35(41 \%)$ & $6(60 \%)$ \\
\hline Stage 2 & $95(42 \%)$ & $31(470 \%)$ & $24(37 \%)$ & $37(43 \%)$ & $3(30 \%)$ \\
\hline Stage 3 & $19(9 \%)$ & $7(11 \%)$ & $4(6 \%)$ & $7(8 \%)$ & $1(10 \%)$ \\
\hline Stage 4 & $7(3 \%)$ & $3(5 \%)$ & $1(2 \%)$ & $3(3 \%)$ & 0 \\
\hline \multicolumn{6}{|l|}{ Tumor status } \\
\hline $\mathrm{T} 1$ & $120(53 \%)$ & $26(40 \%)$ & $38(58 \%)$ & $48(56 \%)$ & $8(80 \%)$ \\
\hline $\mathrm{T} 2$ & $84(37 \%)$ & $29(45 \%)$ & $21(32 \%)$ & $33(38 \%)$ & $1(10 \%)$ \\
\hline T3 & $9(4 \%)$ & $3(5 \%)$ & $2(3 \%)$ & $3(4 \%)$ & $1(10 \%)$ \\
\hline $\mathrm{T} 4$ & $9(4 \%)$ & $6(8 \%)$ & $1(2 \%)$ & $2(2 \%)$ & 0 \\
\hline \multicolumn{6}{|l|}{ Node status } \\
\hline No & $117(52 \%)$ & $28(44 \%)$ & $36(58 \%)$ & $46(53 \%)$ & $7(70 \%)$ \\
\hline N1 & $55(24 \%)$ & $15(23 \%)$ & $18(27 \%)$ & $20(23 \%)$ & $2(20 \%)$ \\
\hline N2 & $11(5 \%)$ & $4(6 \%)$ & $1(2 \%)$ & $5(6 \%)$ & $1(10 \%)$ \\
\hline N3 & $5(2 \%)$ & $2(3 \%)$ & $2(3 \%)$ & $1(1 \%)$ & 0 \\
\hline $\mathrm{Nx}$ & $38(17 \%)$ & $16(25 \%)$ & $8(12 \%)$ & $14(16 \%)$ & 0 \\
\hline \multicolumn{6}{|l|}{ Metastasis status } \\
\hline Mo & $215(95 \%)$ & $62(95 \%)$ & $63(96 \%)$ & $80(93 \%)$ & $8(80 \%)$ \\
\hline M1 & $6(3 \%)$ & $3(5 \%)$ & $1(2 \%)$ & $2(2 \%)$ & 0 \\
\hline $\mathrm{Mx}$ & $5(2 \%)$ & 0 & $1(2 \%)$ & $4(5 \%)$ & $2(20 \%)$ \\
\hline
\end{tabular}

and EGFR ( $P=0.01$ and 0.004 , respectively) and significant negative association between claudin 1 and ER $(P=0.0001)$. In addition, the expression of claudin 4 was positively associated with the expression of EGFR $(P=0.0306)$ and negatively associated with ER $(P=0.0125)$. In contrast to claudins 1 and 4 , claudins 7 and 8 showed a strong direct association with ER expression $(P=0.0028$ and $<0.0001$, respectively) and negative association with CK5/6 $(P=0.0084$ and 0.0072 , respectively $)$ and EGFR expression $(P=0.0001$ and $<0.0001$, respectively).

\section{Expression of Claudins in Different Molecular Subtypes of Breast Cancer}

The relationship between claudins and ER, EGFR, and CK5/6 expression prompted us to assess claudin expression in the different molecular subtypes of breast cancer. A $\chi^{2}$ analysis revealed that the luminal subtype contains significantly more tumors that were claudin 7 positive $(45 \%, P<0.0001)$ and claudin 8 positive $(49 \%, P<0.0001)$ (Table 3$)$. In contrast, high levels of claudin 1 were observed in the basal-like subtype $(63 \%, P=0.0132)$. Claudin 4-positive tumors demonstrated a tendency of association with basal-like tumors (40\%).

\section{Identification of a Claudin-Low Group}

Low levels of expression $(0-1+)$ were detected in $84 \%, 67 \%, 59 \%, 57 \%$, and $61 \%$ of tumors stained for claudins $1,3,4,7$, and 8 , respectively. Low expression of all five claudins was observed in 30 of 226 cases $(14 \%)$ and this group was designated as claudin-low. The majority of the claudin-low group were basal-like cancers ( 23 of $30,77 \%$ ). In contrast, only 1 of $30(3.3 \%)$ tumors was of the luminal phenotype and 6 of $30(20 \%)$ cases were HER2 + $(P<0.0001)$. Within the basal-like subgroup, 9 of 14 $(64 \%)$ of the metaplastic and 14 of $72(19 \%)$ of the non-metaplastic tumors were claudin-low.

\section{Histological Features of Claudin-Low Breast Cancers}

Given the findings that claudin-low tumors are related to the basal-like subtype carcinoma, we more closely examined the histology of these tumors (Figure 3). To quantify our observation, morphological features were rated and mathematically clustered (Figure 4). Two main histological subtypes of claudin-low tumors were identified. The first subtype consisted of 12 cases clustered exclusively with the basal-like group. These tumors showed metaplasia, necrosis, pushing border, as well as syncytial 
and medullary features. The second type contained 15 cases, including basal-like, luminal, and HER2 subtypes, and was characterized by nesting growth pattern with desmoplasia, scar, lymphocytic infiltrate, and associated DCIS. Three cases shared histological features between these two groups.

Of note, the vast majority (6 of $7,86 \%$ ) of metaplastic tumors with chondroid or osseous heterologous elements were claudin-low. In contrast, squamous differentiation was not a feature of claudin-low tumors. Only two of nine claudin-low metaplastic tumors contained squamous components as opposed to four of five claudin non-low metaplastic tumors.

\section{Claudin Expression and Survival}

Univariate analysis of survival was performed to evaluate the impact of conventional prognostic predictors and claudins on patient survival. Kaplan-Meier survival curves were constructed, followed by the log-rank test. Univariate analysis revealed that tumor stage significantly influenced overall patient survival $(P<0.0001$; Figure 5a). Patients with claudin-low tumors had significantly worse recurrent-free survival than the other patients $(P=0.0093$; Figure 5b). Within the basal-like group patients with claudin-low tumors also showed worse recurrent-free survival as opposed to claudin-high tumors $(P=0.11$; Figure 5c). Multivariate analysis of survival revealed that the tumor stage $(P=0.0025)$, and molecular subtype were the only independent predictors of survival $(P=0.0025$ and 0.0345 , respectively). Claudin expression was not an independent predictor of survival by multivariate analysis.

\section{Discussion}

Global gene expression studies identified multiple molecular subtypes of breast cancer, including luminal, HER2-enriched, basal-like, and normal
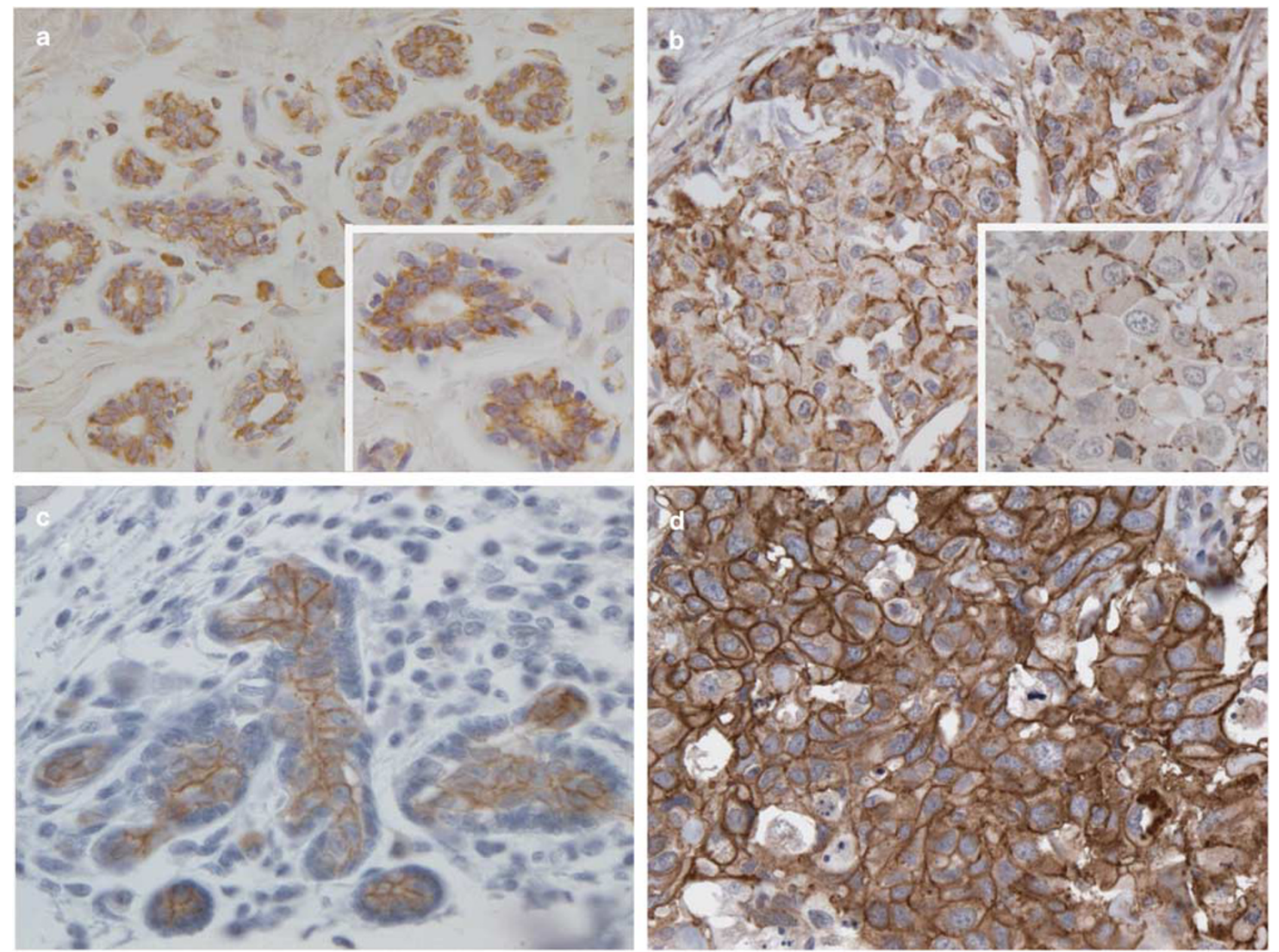

Figure 1 Expression of claudins in normal breast (a, $\mathbf{c}, \mathbf{e}, \mathbf{g}$, and $\mathbf{i}$ ) and invasive ductal carcinoma (b, $\mathbf{d}, \mathbf{f}, \mathbf{h}$, and $\mathbf{j})$. Claudin 1 is expressed in the majority of luminal cells of terminal ductal lobular unit (a) and has scattered pattern in a basal-like carcinoma (b). Claudin 3 is expressed in the apicolateral side of the normal luminal epithelium (c) and the polarity is lost in a basal-like cancer (d). Predominantly apicolateral claudin 4 expression in normal breast (e) and complete circumferential membranous staining in a basal-like carcinoma (f). Claudins 7 and 8 are also apicolateral in the normal gland (g, i) and circumferential membranous in luminal subtype tumors (h, j). 

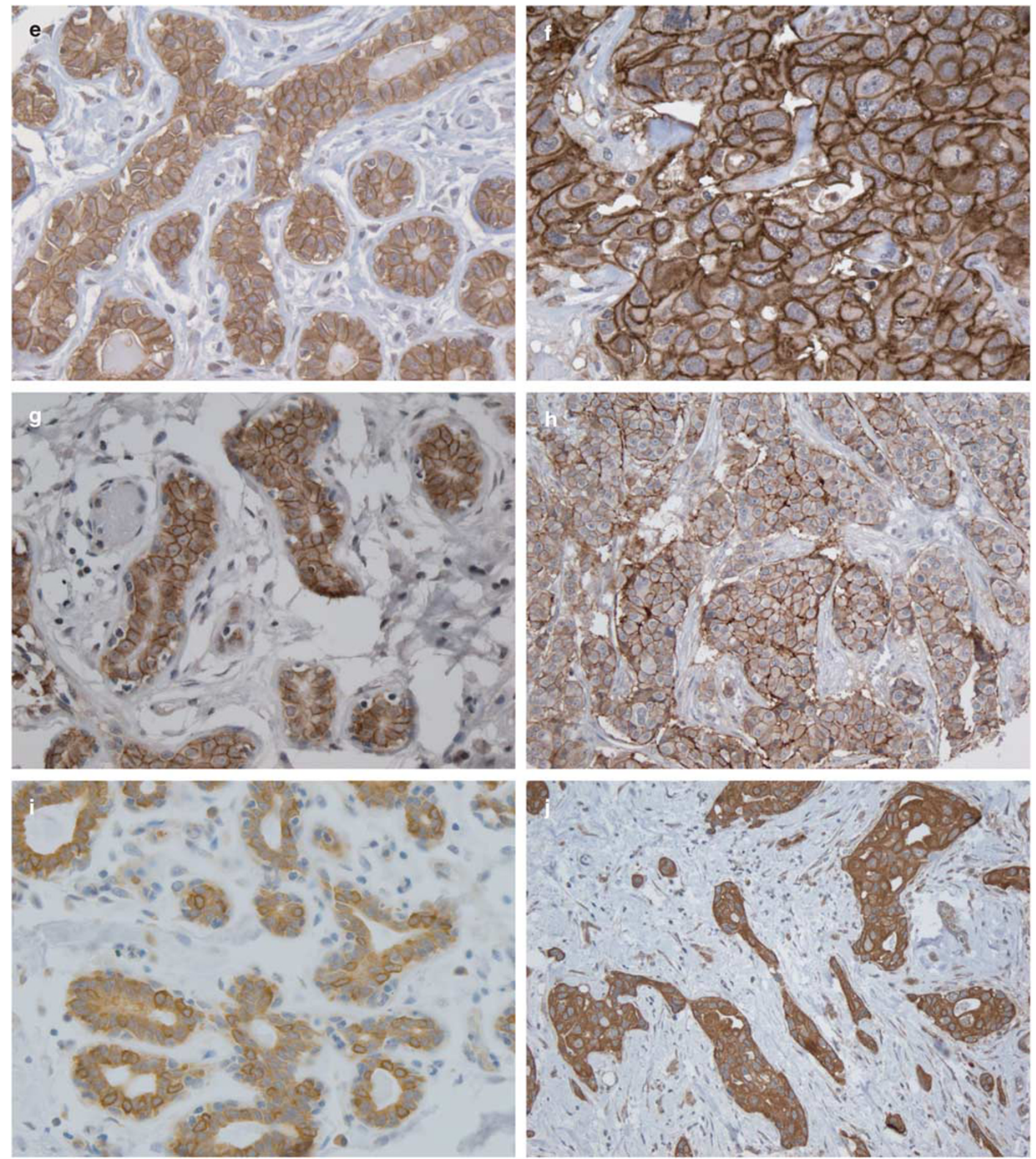

Figure 1 (Continued)

breast-like. The subtypes of these tumors have significant differences in incidence, survival, and response to therapy. Recent genomic studies have identified a novel claudin-low molecular subtype of breast cancer. ${ }^{6}$ In this study, we evaluated the expression patterns of claudins 1, 3, 4, 7, and 8 in different molecular subtypes of grade 3 breast cancer. Selection of claudins evaluated in this study was based on molecular evidence describing low gene expression of claudins 3,4 , and 7 in the claudin-low subtype. ${ }^{9}$ Claudins 1 and 8 were selected based on our previous observations of 


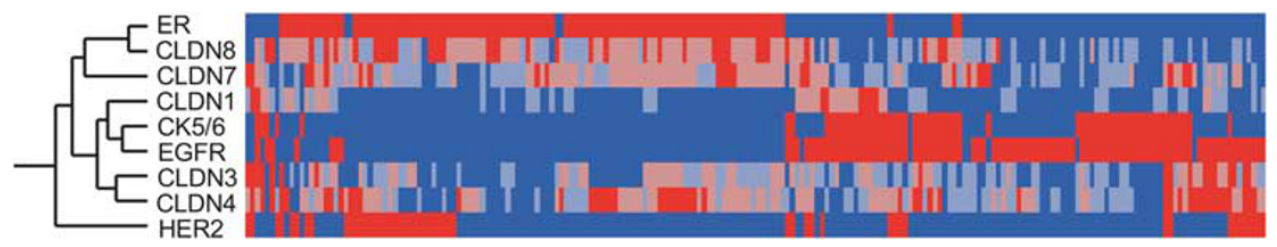

Figure 2 Hierarchical cluster analysis (centroid method with data standardization) of the expressions of claudins 1, 3, 4, 7, and 8 and estrogen receptor (ER), HER2, CK5/6, and epidermal growth factor receptor (EGFR). Each column represents a different tumor, and each row represents a marker. Red: highest expression; blue: lowest expression. The analysis shows that claudins 1, 3, and 4 clustered predominantly with cases positive for basal markers CK5/6 and EGFR, as indicated by short dendrogram branches linking these markers. Claudins 7 and 8 clustered with ER + tumors.

Table 2 Contingency analysis of claudin expression and other markers based on immunohistochemical expression

\begin{tabular}{lcccc}
\hline P-value & ER & HER2 & CK5/6 & EGFR \\
\hline CLDN1 & $\mathbf{0 . 0 0 0 1}$ & NS & 0.01 & 0.004 \\
CLDN3 & N.S. & NS & NS & NS \\
CLDN4 & $\mathbf{0 . 0 1 2 5}$ & NS & NS & 0.0306 \\
CLDN7 & 0.0028 & NS & $\mathbf{0 . 0 0 8 4}$ & $\mathbf{0 . 0 0 0 1}$ \\
CLDN8 & $<0.0001$ & NS & $\mathbf{0 . 0 0 7 2}$ & $<\mathbf{0 . 0 0 0 1}$
\end{tabular}

Bold, negative correlation; italic, positive correlation; NS, no significance $(P>0.05)$.

decreased expression of these tight junction proteins in colonic, gastric, and renal carcinomas. ${ }^{15,16,18}$

Claudins are pivotal proteins in the formation and function of tight junctions. In normal epithelia, the primary functions of the tight junctions are to seal the apical intercellular spaces of glandular epithelia creating a barrier against the paracellular passage of macromolecules and to separate the plasma membrane into the apical and basolateral domains. ${ }^{32,33}$ There are currently at least 24 known members of the claudin family, which are expressed in a tissuespecific pattern in normal epithelia. ${ }^{10,11}$ Neoplastic cells frequently exhibit structural and functional deficiencies in the tight junctions. Disruption of the tight junctions is believed to be one of the processes that occur in carcinogenesis allowing for loss of cellular cohesion, aggressive growth, and differentiation of cancer cells. ${ }^{12,34}$ This hypothesis is supported by the loss of claudin expression in several cancers, including claudin 1 in colorectal carcinoma, ${ }^{15}$ claudins 1 and 7 in prostatic carcinoma, ${ }^{19}$ and claudins 3 and 4 in esophageal carcinoma. ${ }^{35}$ However, some malignant neoplasms do not follow this rule and demonstrate paradoxical overexpression of claudins. Our group has previously reported significantly stronger expression of claudin 4 in colonic and gastric adenocarcinoma as opposed to normal mucosa. ${ }^{15,16}$ Upregulation of claudins 3 and 4 has been observed in ovarian cancer, but not in benign ovarian cystadenomas. ${ }^{36}$ Increased expression of claudins 3 and 4 has been recently shown to contribute to the aggressive behavior of clear-cell and serous papillary endometrial carcinoma. ${ }^{37}$
Table 3 Expression of claudins in different molecular subtypes

\begin{tabular}{|c|c|c|c|c|c|c|}
\hline \multirow[t]{2}{*}{ Total } & & \multicolumn{4}{|c|}{ Molecular subtype } & \multirow[t]{2}{*}{ P-value } \\
\hline & & Luminal & HER2 & Basal-like & Negative & \\
\hline $\begin{array}{l}\text { Claudin } 1 \\
\text { Low } \\
\text { High }\end{array}$ & $\begin{array}{r}186(84 \%) \\
36(16 \%)\end{array}$ & $\begin{array}{c}54(30 \%) \\
3(9 \%)\end{array}$ & $\begin{array}{r}53(30 \%) \\
8(25 \%)\end{array}$ & $\begin{array}{l}62(35 \%) \\
20(63 \%)\end{array}$ & $\begin{array}{l}9(5 \%) \\
1(3 \%)\end{array}$ & 0.0132 \\
\hline $\begin{array}{l}\text { Claudin } 3 \\
\text { Low } \\
\text { High }\end{array}$ & $\begin{array}{r}148(67 \%) \\
73(33 \%)\end{array}$ & $\begin{array}{l}38(27 \%) \\
19(28 \%)\end{array}$ & $\begin{array}{l}41(29 \%) \\
21(31 \%)\end{array}$ & $\begin{array}{l}58(41 \%) \\
24(36 \%)\end{array}$ & $\begin{array}{l}5(4 \%) \\
3(5 \%)\end{array}$ & 0.9103 \\
\hline $\begin{array}{l}\text { Claudin } 4 \\
\text { Low } \\
\text { High }\end{array}$ & $\begin{array}{r}128(59 \%) \\
90(41 \%)\end{array}$ & $\begin{array}{l}38(31 \%) \\
18(21 \%)\end{array}$ & $\begin{array}{l}32(26 \%) \\
29(34 \%)\end{array}$ & $\begin{array}{l}47(39 \%) \\
34(40 \%)\end{array}$ & $\begin{array}{l}4(3 \%) \\
5(6 \%)\end{array}$ & 0.2944 \\
\hline $\begin{array}{l}\text { Claudin } 7 \\
\text { Low } \\
\text { High }\end{array}$ & $\begin{array}{r}115(57 \%) \\
87(43 \%)\end{array}$ & $\begin{array}{l}18(17 \%) \\
37(45 \%)\end{array}$ & $\begin{array}{l}30(28 \%) \\
21(25 \%)\end{array}$ & $\begin{array}{l}58(54 \%) \\
19(23 \%)\end{array}$ & $\begin{array}{l}1(1 \%) \\
6(7 \%)\end{array}$ & $<0.0001$ \\
\hline $\begin{array}{l}\text { Claudin } 8 \\
\text { Low } \\
\text { High }\end{array}$ & $\begin{array}{r}129(61 \%) \\
82(39 \%)\end{array}$ & $\begin{array}{l}20(17 \%) \\
39(49 \%)\end{array}$ & $\begin{array}{l}27(23 \%) \\
25(31 \%)\end{array}$ & $\begin{array}{l}66(56 \%) \\
15(19 \%)\end{array}$ & $\begin{array}{l}6(5 \%) \\
1(1 \%)\end{array}$ & $<0.0001$ \\
\hline $\begin{array}{l}\text { Claudins } \\
\text { All low } \\
\text { Non-low }\end{array}$ & $\begin{array}{r}30(16 \%) \\
190(84 \%)\end{array}$ & $\begin{array}{c}1(3 \%) \\
64(34 \%)\end{array}$ & $\begin{array}{r}6(20 \%) \\
59(31 \%)\end{array}$ & $\begin{array}{l}23(77 \%) \\
63(33 \%)\end{array}$ & $\begin{array}{c}0 \\
10(5 \%)\end{array}$ & $<0.0001$ \\
\hline
\end{tabular}

Our findings demonstrate that the expression of claudins in invasive ductal carcinoma of the breast is not only tissue specific but is also unique in particular molecular subtypes of breast cancer. Luminal cancers appear to exhibit ER +/claudins 7, 8 high/claudins 1, 4 low signature, and therefore claudins 7 and 8 may be designated as 'luminal'. In contrast, basal-like cancers demonstrate ER-I HER2-/claudins 1, 4 high/claudins 7, 8 low phenotype, and claudins 1 and 4 may be considered as 'basal' claudins. To date, only a few studies have addressed the role of claudins in breast carcinoma. Studies of the expression of claudins 3 and 4 in invasive ductal carcinoma remain controversial. In one of the early studies, Soini ${ }^{38}$ observed strong expression of claudins 3 and 4 in invasive ductal carcinoma; however, he did not find an association between the expression of claudins 3 and 4 and tumor grade or ER status. Tokes et $a l^{39}$ reported significant loss of claudin 4 mRNA and protein expression in the majority of grade 1 ductal carcinomas, but increased expression in grade 2 and 3 tumors Our findings of an association of the expression of claudins 1 and 4 with ER - tumors are in complete agreement with several recent 

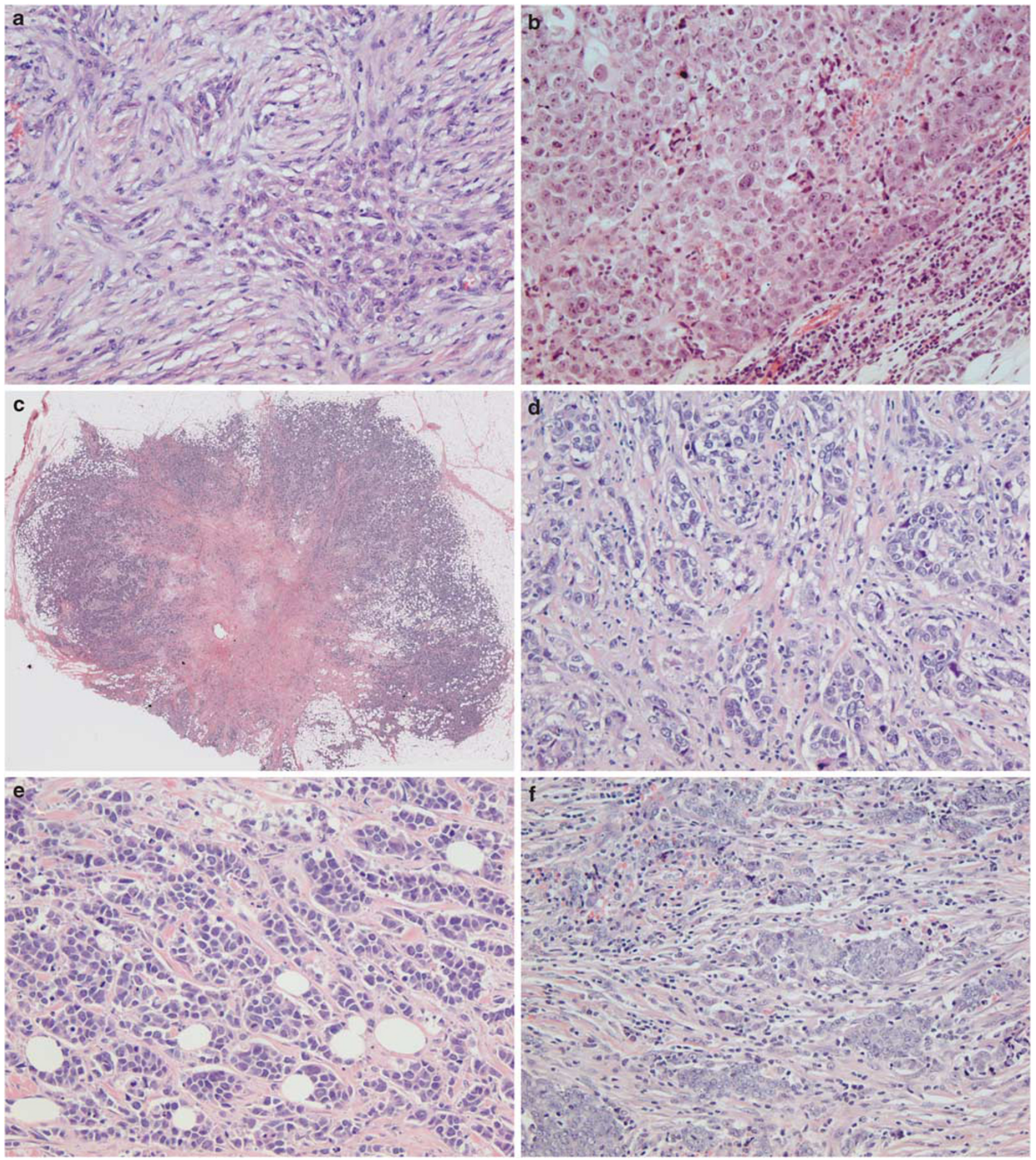

Figure 3 Histological features of claudin-low breast cancer. (a) Metaplastic cancer, (b) medullary growth pattern, (c) central scar, (d) desmoplastic stromal reaction, (e) nesting growth pattern, and (f) lymphocytic infiltrate.

reports. ${ }^{26,27}$ Lanigan et $a l^{26}$ described the association of increased expression of claudin 4 with high tumor grade, ER - tumor status, and poor prognosis.(26) Blanchard et $a l^{27}$ demonstrated correlation of claudins 1 and 4 with the basal-like subtype. Similar findings on overexpression of claudin 4 in basal-like subtype of breast cancer have been reported by Kulka et al. ${ }^{28}$

Only a few studies have examined the expression of claudin 7 in breast carcinomas. Kominsky et $a l^{22}$ reported loss of claudin 7 in high-grade invasive ductal carcinoma. Upon analyzing data of claudin 7 


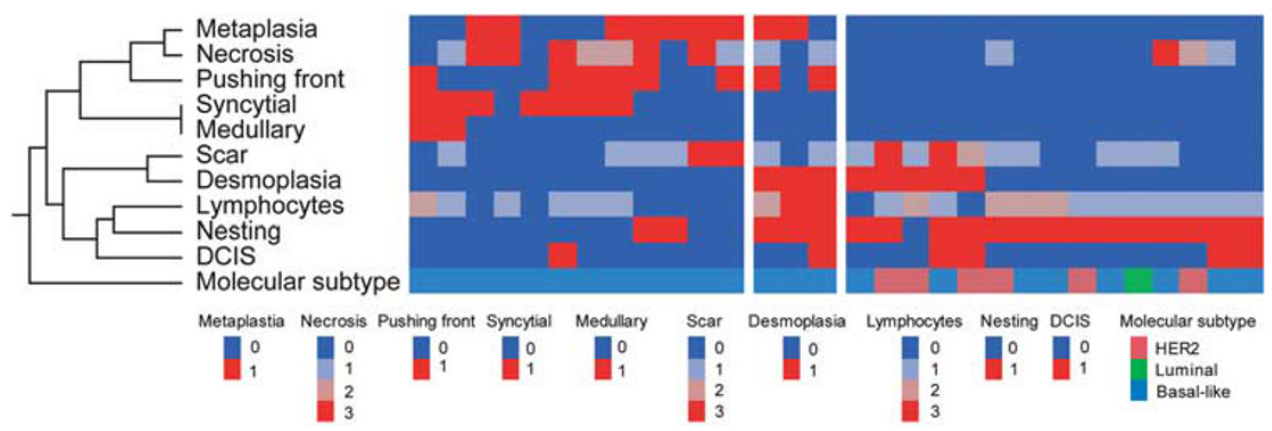

Figure 4 Hierarchical cluster analysis (complete method with data standardization) of histological features of claudin-low cancer. Each column represents a different tumor, and each row represents a histological feature. Red: highest expression; blue: lowest expression. The analysis shows two main groups.
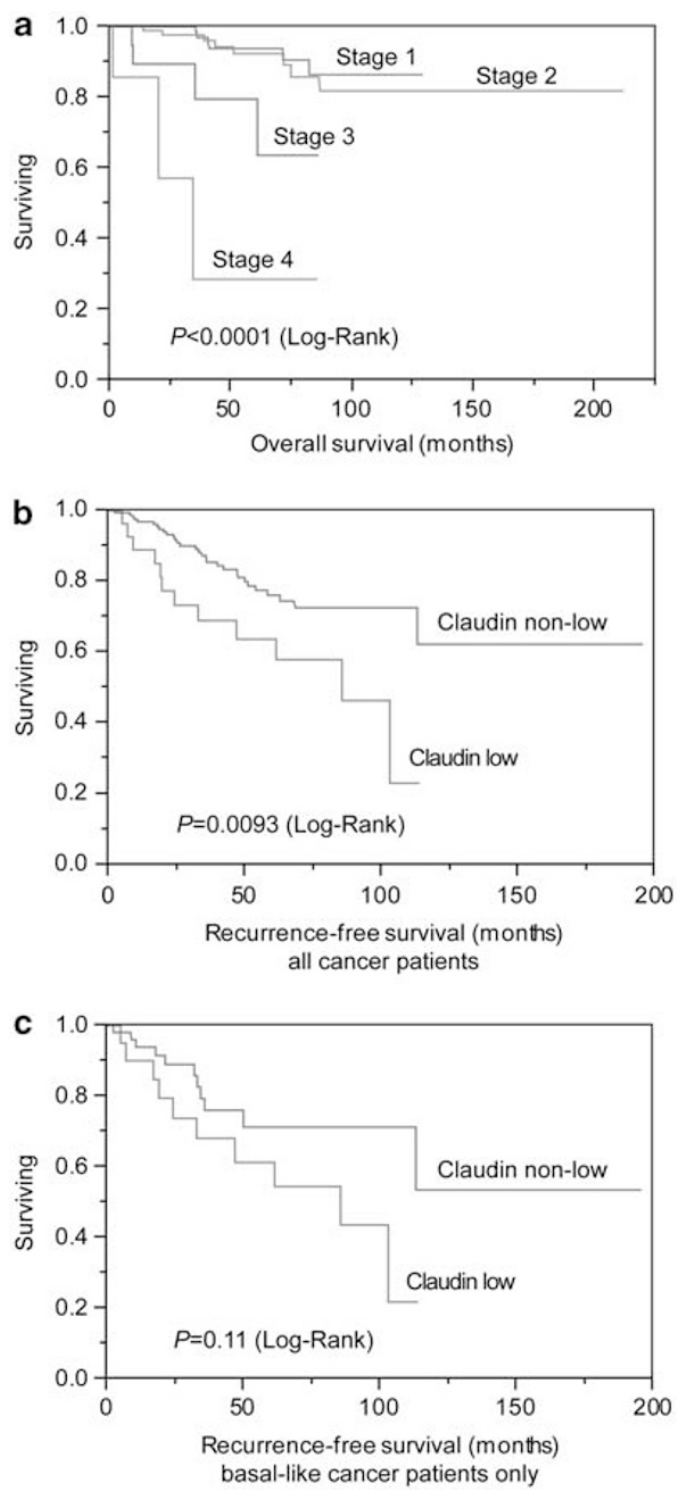

Figure 5 Analysis of overall (a) and recurrence-free survival (b,c) in grade 3 invasive ductal carcinoma by stage (a), claudin-low expression in the entire cohort (b), and claudin-low expression in basal-like subtype only (c).
mRNA expression quantitated by real-time PCR from this study, in which breast cancer cell lines were used, we found that all ER - cell lines showed no detectable claudin 7 mRNA, wheres all ER + cell lines showed detectable expression of claudin 7. In parallel with our data, reduced immunohistochemical expression of claudin 7 was associated with ER - breast carcinomas, ${ }^{23}$ and was less frequent in the triple-negative group. ${ }^{24}$ Here we present, for the first time, immunohistochemical evidence of the expression of claudin 8 in invasive ductal carcinoma and demonstrated its association with the ER + luminal subtype of breast cancer.

In this study, we defined a group of tumors negative for all five claudins tested and demonstrated that this claudin-low group is strongly associated with basal-like breast cancer and metaplastic cancer. Claudin-low subtype of breast cancer was identified in 2007 by gene expression analysis of breast tumors. ${ }^{6}$ This group is characterized by low expression of genes involved in tight junctions and cell-cell adhesion, including claudins 3,4 , and $7 .^{9}$ The claudin-low group is characterized by enrichment of epithelial-to-mesenchymal transition markers, immune response genes, and cancer stem cell markers. In contrast to other molecular subtypes of breast cancer, claudin-low tumors have been less studied. Recently, Prat et $a l^{9}$ comprehensively characterized this subtype at the molecular level by hierarchical clustering of approximately 1900 intrinsic genes and found that this group comprises approximately $12 \%$ of breast cancers. Our data show similar frequencies of claudin-low group (13\%). One would expect higher frequencies in this study group containing only high-grade tumors; however, our criteria required all five claudins to be low, thereby decreasing the number of claudin-low cases. As the expression of more claudin subtypes are evaluated in breast cancer, the definition of the claudin low variant will likely need to be modified.

Our results provide evidence that the basallike subtype of breast cancer is a heterogeneous group and may be further subdivided into subgroup 
with loss of claudin expression (claudin-low) and a subgroup with gain of claudin expression (claudin-high). Recently, Myal et al ${ }^{40}$ discussed the significance of a subset of ER - breast cancers, which express high levels of claudin 1 and proposed a possible 'claudin-high' subset of breast cancer. Our results demonstrate that claudin 1-high subgroup is a relatively frequent phenomenon in basal-like breast cancer. In both scenarios of claudin loss or gain, it appears that abnormal claudin expression is associated with more aggressive tumor behavior.

In summary, we demonstrated a differential claudin expression in the four molecular subtypes of invasive ductal carcinoma and proposed 'luminal' and 'basal' claudins. Molecular subtypes of breast cancer are a heterogeneous group and can be further subdivided into a claudin-low group. Claudin-low subtype is a frequent phenomenon in metaplastic and basal-like breast cancer and appears to be a strong predictor of disease recurrence.

\section{Acknowledgements}

This study was supported by the Brown University Pathology Pilot Project Funds for Translational Research and the Molecular Pathology Core of the COBRE Center for Cancer Research Development, funded by the National Institute of General Medical Sciences of the National Institutes of Health under Award Number P20GM103421. The content is solely the responsibility of the authors and does not necessarily represent the official views of the National Institutes of Health.

\section{Disclosure/conflict of interest}

The authors declare no conflict of interest.

\section{References}

1 Siegel R, Naishadham D, Jemal A. Cancer statistics, 2012. CA Cancer J Clin 2012;62:10-29.

2 Perou CM, Sorlie T, Eisen MB, et al. Molecular portraits of human breast tumours. Nature 2000;406:747-752.

3 Sorlie T, Perou CM, Tibshirani R, et al. Gene expression patterns of breast carcinomas distinguish tumor subclasses with clinical implications. Proc Natl Acad Sci USA 2001;98:10869-10874.

4 Nielsen TO, Hsu FD, Jensen K, et al. Immunohistochemical and clinical characterization of the basal-like subtype of invasive breast carcinoma. Clin Cancer Res 2004;10:5367-5374.

5 Carey LA, Perou CM, Livasy CA, et al. Race, breast cancer subtypes, and survival in the Carolina Breast Cancer Study. JAMA 2006;295:2492-2502.

6 Herschkowitz JI, Simin K, Weigman VJ, et al. Identification of conserved gene expression features between murine mammary carcinoma models and human breast tumors. Genome Biol 2007;8:R76.
7 Hennessy BT, Gonzalez-Angulo AM, Stemke-Hale K, et al. Characterization of a naturally occurring breast cancer subset enriched in epithelial-to-mesenchymal transition and stem cell characteristics. Cancer Res 2009;69:4116-4124.

8 Taube JH, Herschkowitz JI, Komurov K, et al. Core epithelial-to-mesenchymal transition interactome gene-expression signature is associated with claudinlow and metaplastic breast cancer subtypes. Proc Natl Acad Sci USA 2010;107:15449-15454.

9 Prat A, Parker JS, Karginova O, et al. Phenotypic and molecular characterization of the claudin-low intrinsic subtype of breast cancer. Breast Cancer Res 2010;12:R68.

10 Morita K, Furuse M, Fujimoto K, et al. Claudin multigene family encoding four-transmembrane domain protein components of tight junction strands. Proc Natl Acad Sci USA 1999;96:511-516.

11 Tsukita S, Furuse M, Itoh M. Multifunctional strands in tight junctions. Nat Rev Mol Cell Biol 2001;2: 285-293.

12 Morin PJ. Claudin proteins in human cancer: promising new targets for diagnosis and therapy. Cancer Res 2005;65:9603-9606.

13 Soini Y. Expression of claudins 1, 2, 3, 4, 5 and 7 in various types of tumours. Histopathology 2005;46:551-560.

14 Hewitt KJ, Agarwal R, Morin PJ. The claudin gene family: expression in normal and neoplastic tissues. BMC Cancer 2006;6:186.

15 Resnick MB, Konkin T, Routhier J, et al. Claudin-1 is a strong prognostic indicator in stage II colonic cancer: a tissue microarray study. Mod Pathol 2005;18:511-518.

16 Resnick MB, Gavilanez M, Newton E, et al. Claudin expression in gastric adenocarcinomas: a tissue microarray study with prognostic correlation. Hum Pathol 2005;36:886-892.

17 Michl P, Barth C, Buchholz M, et al. Claudin-4 expression decreases invasiveness and metastatic potential of pancreatic cancer. Cancer Res 2003;63: 6265-6271.

18 Lechpammer M, Resnick MB, Sabo E, et al. The diagnostic and prognostic utility of claudin expression in renal cell neoplasms. Mod Pathol 2008;21: 1320-1329.

19 Sheehan GM, Kallakury BV, Sheehan CE, et al. Loss of claudins- 1 and -7 and expression of claudins- 3 and -4 correlate with prognostic variables in prostatic adenocarcinomas. Hum Pathol 2007;38:564-569.

20 Choi YL, Kim J, Kwon MJ, et al. Expression profile of tight junction protein claudin 3 and claudin 4 in ovarian serous adenocarcinoma with prognostic correlation. Histol Histopathol 2007;22:1185-1195.

21 Jung JH, Jung CK, Choi HJ, et al. Diagnostic utility of expression of claudins in non-small cell lung cancer: different expression profiles in squamous cell carcinomas and adenocarcinomas. Pathol Res Pract 2009;205:409-416.

22 Kominsky SL, Argani P, Korz D, et al. Loss of the tight junction protein claudin-7 correlates with histological grade in both ductal carcinoma in situ and invasive ductal carcinoma of the breast. Oncogene 2003;22:2021-2033.

23 Park D, Kåresen R, Axcrona U, et al. Expression pattern of adhesion molecules (E-cadherin, alpha-, beta-, gamma-catenin and claudin-7), their influence on survival in primary breast carcinoma, and their 
corresponding axillary lymph node metastasis. APMIS 2007;115:52-65.

24 Bernardi MA, Logullo AF, Pasini FS, et al. Prognostic significance of CD24 and claudin-7 immunoexpression in ductal invasive breast cancer. Oncol Rep 2012;27:28-38.

25 Kominsky SL, Vali M, Korz D, et al. Clostridium perfringens enterotoxin elicits rapid and specific cytolysis of breast carcinoma cells mediated through tight junction proteins claudin 3 and 4. Am J Pathol 2004;164:1627-1633.

26 Lanigan F, McKiernan E, Brennan DJ, et al. Increased claudin-4 expression is associated with poor prognosis and high tumour grade in breast cancer. Int J Cancer 2009;124:2088-2097.

27 Blanchard AA, Skliris GP, Watson PH, et al. Claudins 1, 3, and 4 protein expression in ER negative breast cancer correlates with markers of the basal phenotype. Virchows Arch 2009;454:647-656.

28 Kulka J, Szász AM, Németh Z, et al. Expression of tight junction protein claudin-4 in basal-like breast carcinomas. Pathol Oncol Res 2009;15:59-64.

29 Elston CW, Ellis IO. Pathological prognostic factors in breast cancer. I. The value of histological grade in breast cancer: experience from a large study with longterm follow-up. Histopathology 1991;19:403-410.

30 Edge SB, Byrd DR, Compton CC, et al. (eds). AJCC cancer staging manual. Springer-Verlag: New York, 2010, pp 347-378.

31 Wolff AC, Hammond ME, Schwartz JN, et al. American Society of Clinical Oncology/College of American Pathologists guideline recommendations for human epidermal growth factor receptor 2 testing in breast cancer. Arch Pathol Lab Med 2007;131:18-43.

32 Madara JL. Regulation of the movement of solutes across tight junctions. Annu Rev Physiol 1998;60:143-159.

33 Tsukita S, Furuse M. The structure and function of claudins, cell adhesion molecules at tight junctions. Ann N Y Acad Sci 2000;915:129-135.

34 Escudero-Esparza A, Jiang WG, Martin TA. The Claudin family and its role in cancer and metastasis. Front Biosci 2011;16:1069-1083.

35 Takala H, Saarnio J, Wiik H, Soini Y. Claudins 1, 3, 4, 5 and 7 in esophageal cancer: loss of claudin 3 and 4 expression is associated with metastatic behavior. APMIS 2007;115:838-847.

36 Rangel LB, Agarwal R, D’Souza T, et al. Tight junction proteins claudin-3 and claudin-4 are frequently overexpressed in ovarian cancer but not in ovarian cystadenomas. Clin Cancer Res 2003;9:2567-2575.

37 Konecny GE, Agarwal R, Keeney GA, et al. Claudin-3 and claudin-4 expression in serous papillary, clearcell, and endometrioid endometrial cancer. Gynecol Oncol 2008;109:263-269.

38 Soini Y. Claudins 2, 3, 4, and 5 in Paget's disease and breast carcinoma. Hum Pathol 2004;35:1531-1536.

39 Tokés AM, Kulka J, Paku S, et al. Claudin-1, -3 and -4 proteins and mRNA expression in benign and malignant breast lesions: a research study. Breast Cancer Res 2005; 7:R296-305.

40 Myal Y, Leygue E, Blanchard AA. Claudin 1 in breast tumorigenesis: revelation of a possible novel 'claudin high' subset of breast cancers. J Biomed Biotechnol 2010;2010:956897. 